\title{
A Robust Spline Collocation Method for Pricing American Put Options
}

\author{
Zhongdi Cen $(\mathbb{D}$, Anbo Le $(\mathbb{D}$, and Aimin Xu \\ Institute of Mathematics, Zhejiang Wanli University, Ningbo 315100, Zhejiang, China \\ Correspondence should be addressed to Anbo Le; anbole7@hotmail.com
}

Received 29 January 2019; Revised 27 March 2019; Accepted 18 April 2019; Published 2 May 2019

Academic Editor: Xiaohua Ding

Copyright (C) 2019 Zhongdi Cen et al. This is an open access article distributed under the Creative Commons Attribution License, which permits unrestricted use, distribution, and reproduction in any medium, provided the original work is properly cited.

\begin{abstract}
In this paper a robust numerical method is proposed for pricing American put options. The Black-Scholes differential operator in the original form is discretized by using a quadratic spline collocation method on a piecewise uniform mesh for the spatial discretization and the implicit Euler scheme for the time discretization. The position of collocation points is chosen so that the spline difference operator satisfies the discrete maximum principle, which guarantees that the scheme is maximum-norm stable. The error estimation is derived by applying the maximum principle to the discrete linear complementarity problem in two mesh sets. It is proved that the scheme is second-order convergent with respect to the spatial variable and first-order convergent with respect to the time variable. Numerical results demonstrate that the scheme is stable and accurate.
\end{abstract}

\section{Introduction}

The American option is an important financial instrument that gives the holder the right, but not obligation, to buy (call option) or sell (put option) an asset at any time prior to its maturity date. One way to price American options is to solve a linear complementarity problem involving the Black-Scholes differential operator [1]. Since this complementarity problem is, in general, not analytically solvable, numerical methods are required to obtain the approximate solution.

The spline approximation methods have become interesting and very promising in solving differential equations due to their flexibility in practical applications. The spline solution has its own advantages, for example, once the solution has been computed, the information required for the spline interpolation between mesh points is easy to obtain. A few papers have used the spline approximation methods to solve option pricing problems. Khabir and Patidar [2] applied a Bspline collocation method to solve the heat equation which is obtained from the Black-Scholes equation by an Euler transformation. Kadalbajoo et al. [3,4] used cubic B-spline collocation methods for the Euler transformed generalized Black-Scholes equation. Mohammadi [5] developed a quintic B-spline collocation method for solving the generalized
Black-Scholes equation governing option pricing. Christara and Leung [6] derived a quadratic spline collocation method on a uniform mesh in the pricing problem under a finite activity jump-diffusion model. Rashidinia and Jamalzadeh [7] proposed a modified B-spline collocation approach for pricing American style Asian options.

In this paper we adopt a quadratic spline collocation method on a piecewise uniform mesh to solve the continuous linear complementarity problem arising from American put option pricing. The Black-Scholes differential operator in the original form is discretized. By applying the technique of Surla et al. $[8,9]$, the position of collocation points is chosen so that the spline difference operator on a piecewise uniform mesh satisfies the discrete maximum principle, which guarantees that the discretization scheme is maximum-norm stable. The error estimation is derived by applying the maximum principle to the discrete linear complementarity problem in two mesh sets. It is proved that the convergence order of the scheme is second-order with respect to the spatial variable and first-order with respect to the time variable. The scheme examined in the present paper overcomes the difficulty in the derivation of the error estimation for the linear complementarity problem under the difference schemes. Numerical results are presented to support these theoretical results. 
The outline of the paper is as follows. In Section 2 the continuous linear complementarity problem for pricing American put options is given. In Section 3 the spline difference scheme is derived. In Section 4 the stability and error analysis for the spline difference scheme are proved. In Section 5 numerical experiments are carried out. Finally conclusion and discussion are indicated in Section 6.

\section{The Continuous Problem}

It is well known that the value of an American put option $v(x, t)$ satisfies the following continuous linear complementarity problem $[1,10]$ :

$$
\begin{aligned}
L v(x, t) \geq 0, & \\
v(x, t)-g(x) \geq 0, & \\
x>0 \text { and } t \in[0, T), & \text { and } t \in[0, T], \\
L v(x, t) \cdot[v(x, t)-g(x)] & =0, \\
v(x, T) & =g(x), \quad x \geq 0 \text { and } t=T, \\
v(0, t) & =E, \\
v(x, t) & \longrightarrow 0, \\
x \longrightarrow+\infty \text { and } t \in[0, T], &
\end{aligned}
$$

where $L$ represents the Black-Scholes differential operator defined by

$$
L v(x, t) \equiv-\frac{\partial v}{\partial t}-\frac{1}{2} \sigma^{2} x^{2} \frac{\partial^{2} v}{\partial x^{2}}-(r-d) x \frac{\partial v}{\partial x}+r v,
$$

$x$ is the underlying asset price, $t$ is the time, $\sigma$ is the volatility of the underlying asset price, $r$ is the risk-free interest rate, $d$ is the continuous dividend rate, $E$ is the strike price, $T$ is the maturity date, and $g(x)$ is the payoff function given by

$$
g(x)=\max (E-x, 0) .
$$

Here we assume that $r>d$.

The infinite domain $(0,+\infty)$ needs to be truncated into a bounded domain $(0, X)$ for solving the problem by the numerical method, where $X$ is taken as $X=4 E$ based on Willmott et al's estimate [1]. The boundary condition at $x=X$ is set to be $v(X, t)=0$. A detailed discussion of the choice of the linear boundary conditions can be found in [11]. Normally, the error in the computed option price due to the domain truncation is negligible [12]. Therefore, in the remaining of this paper we will focus on solving the following linear complementarity problem

$$
\begin{aligned}
& L v(x, t) \geq 0, \\
& (x, t) \in(0, X) \times(0, T), \\
& v(x, t)-g(x) \geq 0, \\
& (x, t) \in(0, X) \times[0, T], \\
& L v(x, t) \cdot[v(x, t)-g(x)]=0, \\
& (x, t) \in(0, X) \times[0, T], \\
& v(x, T)=g(x), \quad x \in[0, X], \\
& v(0, t)=E, \\
& v(X, t)=0,
\end{aligned}
$$

\section{Discretization}

Since the Black-Scholes differential operator at $x=0$ is degenerative, the Euler transformation is usually used to remove the singularity of the differential operator at $x=$ 0 (corresponding to $-\infty$ in the transformed domain); see, e.g., Schwartz [13], Tangman et al. [14], and Zhao et al. [15]. However, this will result in additional computational errors due to the truncation on the left-hand side of the domain. Furthermore, the originally mesh points will concentrate around $x=0$ inappropriately by using the uniform mesh on the transformed interval. In this paper we will discretize the Black-Scholes equation in the original form, but with a quadratic spline collocation method for the spatial discretization and the implicit Euler scheme for the temporal discretization. It should be remarked that if the commonly quadratic spline collocation method is adopted on a uniform mesh for the original Black-Scholes equation, the stability of the scheme could not be guaranteed. The analogous problems have been discussed in $[16,17]$.

For the spatial discretization, a piecewise uniform mesh $\Omega^{N}$ is constructed to guarantee the stability of the discretization scheme:

$$
x_{i}= \begin{cases}h & i=1, \\ h\left[1+\frac{\sigma^{2}}{r-d}(i-1)\right] & i=2, \cdots, N,\end{cases}
$$

where

$$
h=\frac{X}{1+\left(\sigma^{2} /(r-d)\right)(N-1)} .
$$

For the temporal discretization, a uniform mesh $\Omega^{K}$ on $[0, T]$ with $K$ mesh elements is used. Then the domain $\Omega \equiv$ $(0, X) \times(0, T)$ is divided into the piecewise uniform mesh 
$\Omega^{N \times K} \equiv \Omega^{N} \times \Omega^{K}$. The mesh sizes $h_{i}=x_{i}-x_{i-1}$ and $\Delta t=t_{j}-t_{j-1}$, respectively, satisfy

$$
h_{i}= \begin{cases}h & i=1, \\ \frac{\sigma^{2}}{r-d} h & i=2, \cdots, N,\end{cases}
$$

and

$$
\Delta t=\frac{T}{K}, \quad j=1, \cdots, K .
$$

It will be shown that the matrix associated with the spline difference operator on the above piecewise uniform mesh is an M-matrix.

The implicit Euler scheme on $\Omega^{K}$ is used for the time discretization to get the following linear complementarity problem:

$$
\begin{gathered}
\widetilde{L} v^{j}(x) \geq \frac{v^{j+1}(x)}{\Delta t}, \\
x \in(0, X), \\
v^{j}(x)-g(x) \geq 0, \\
x \in(0, X), \\
{\left[\widetilde{L} v^{j}(x)-\frac{v^{j+1}(x)}{\Delta t}\right] \cdot\left[v^{j}(x)-g(x)\right]=0,} \\
x \in(0, X), \\
v^{K}(x)=g(x), \\
x \in[0, X], \\
v^{j}(0)=E, \\
v^{j}(X)=0,
\end{gathered}
$$

where

$$
\begin{aligned}
\widetilde{L} v^{j}(x)= & -\frac{1}{2} \sigma^{2} x^{2} \frac{d^{2} v^{j}}{d x^{2}}-(r-d) x \frac{d v^{j}}{d x} \\
& +\left(r+\frac{1}{\Delta t}\right) v^{j}(x),
\end{aligned}
$$

and $v^{j}(x)$ denotes the approximation of the exact solution $v(x, t)$ at $j$-th time level.

The solution $v^{j}(x)$ of the problem (18)-(22) is approximated by a quadratic spline function $u(x) \in C^{1}([0, X])$ which on each subinterval $\left[x_{i}, x_{i+1}\right]$ has the following form:

$$
\begin{aligned}
u(x)=u_{i}+\left(x-x_{i}\right) u_{i}^{\prime}+\frac{1}{2}\left(x-x_{i}\right)^{2} u_{i}^{\prime \prime}, & \\
x & \in\left[x_{i}, x_{i+1}\right] .
\end{aligned}
$$

The collocation points are chosen in a nonstandard way as that in $[8,9]$ :

$$
\begin{array}{ll}
\xi_{i}=\alpha_{1, i} x_{i-1}+\left(1-\alpha_{1, i}\right) x_{i} & \text { on }\left[x_{i-1}, x_{i}\right], \\
\eta_{i}=\alpha_{2, i} x_{i}+\left(1-\alpha_{2, i}\right) x_{i+1} & \text { on }\left[x_{i}, x_{i+1}\right]
\end{array}
$$

for $1 \leq i<N$, where $0<\alpha_{1, i}, \alpha_{2, i}<1$. Then the collocation equations are defined by

$$
\begin{aligned}
& -\frac{1}{2} \sigma^{2} \xi_{i}^{2} u^{\prime \prime}\left(\xi_{i}\right)-(r-d) \xi_{i} u^{\prime}\left(\xi_{i}\right)+\left(r+\frac{1}{\Delta t}\right) u\left(\xi_{i}\right) \\
& \quad=\widetilde{L} u\left(\xi_{i}\right), \quad \xi_{i} \in\left[x_{i-1}, x_{i}\right], \\
& -\frac{1}{2} \sigma^{2} \eta_{i}^{2} u^{\prime \prime}\left(\eta_{i}\right)-(r-d) \eta_{i} u^{\prime}\left(\eta_{i}\right)+\left(r+\frac{1}{\Delta t}\right) u\left(\eta_{i}\right) \\
& =\widetilde{L} u\left(\eta_{i}\right), \quad \eta_{i} \in\left[x_{i}, x_{i+1}\right] .
\end{aligned}
$$

We can obtain $u^{\prime \prime}\left(\xi_{i}\right)=u_{i-1}^{\prime \prime}, u^{\prime \prime}\left(\eta_{i}\right)=u_{i}^{\prime \prime}$ from the quadratic spline function (24). Since $\xi_{i}-x_{i-1}=h_{i}\left(1-\alpha_{1, i}\right)$, $\eta_{i}-x_{i}=h_{i+1}\left(1-\alpha_{2, i}\right)$, we have

$$
\begin{aligned}
u\left(\xi_{i}\right)= & u_{i-1}+h_{i}\left(1-\alpha_{1, i}\right) u_{i-1}^{\prime} \\
& +\frac{1}{2} h_{i}^{2}\left(1-\alpha_{1, i}\right)^{2} u_{i-1}^{\prime \prime}, \\
u\left(\eta_{i}\right)= & u_{i}+h_{i+1}\left(1-\alpha_{2, i}\right) u_{i}^{\prime}+\frac{1}{2} h_{i+1}^{2}\left(1-\alpha_{2, i}\right)^{2} u_{i}^{\prime \prime}, \\
u^{\prime}\left(\xi_{i}\right)= & u_{i-1}^{\prime}+h_{i}\left(1-\alpha_{1, i}\right) u_{i-1}^{\prime \prime}, \\
u^{\prime}\left(\eta_{i}\right)= & u_{i}^{\prime}+h_{i+1}\left(1-\alpha_{2, i}\right) u_{i}^{\prime \prime} .
\end{aligned}
$$

Substituting the above expressions for $u\left(\eta_{i}\right), u^{\prime}\left(\eta_{i}\right)$, and $u^{\prime \prime}\left(\eta_{i}\right)$ into (27) on the interval $\left[x_{i}, x_{i+1}\right]$ with

$$
\begin{aligned}
& u_{i+1}=u_{i}+h_{i+1} u_{i}^{\prime}+\frac{1}{2} h_{i+1}^{2} u_{i}^{\prime \prime}, \\
& u_{i+1}^{\prime}=u_{i}^{\prime}+h_{i+1} u_{i}^{\prime \prime},
\end{aligned}
$$

we have

$$
\begin{aligned}
u_{i}^{\prime} & =\frac{\left(u_{i+1}-u_{i}\right) Q_{i}+(r+1 / \Delta t) h_{i+1}^{2} u_{i}-h_{i+1}^{2} \widetilde{L} u\left(\eta_{i}\right)}{h_{i+1} P_{i}}, \\
u_{i+1}^{\prime} & \\
= & \frac{2\left(u_{i+1}-u_{i}\right)}{h_{i+1}} \\
& -\frac{\left(u_{i+1}-u_{i}\right) Q_{i}+(r+1 / \Delta t) h_{i+1}^{2} u_{i}-h_{i+1}^{2} \widetilde{L} u\left(\eta_{i}\right)}{h_{i+1} P_{i}},
\end{aligned}
$$

where

$$
\begin{aligned}
Q_{i}= & -\sigma^{2} \eta_{i}^{2}-2(r-d) \eta_{i} h_{i+1}\left(1-\alpha_{2, i}\right) \\
& +\left(r+\frac{1}{\Delta t}\right) h_{i+1}^{2}\left(1-\alpha_{2, i}\right)^{2},
\end{aligned}
$$




$$
\begin{aligned}
P_{i}= & -\sigma^{2} \eta_{i}^{2}+(r-d) \eta_{i} h_{i+1}\left(-1+2 \alpha_{2, i}\right) \\
& -\left(r+\frac{1}{\Delta t}\right) h_{i+1}^{2} \alpha_{2, i}\left(1-\alpha_{2, i}\right) .
\end{aligned}
$$

Using the same technique on the interval $\left[x_{i-1}, x_{i}\right]$ with the collocation equation (26) and replacing $i$ by $i-1$ in (31), we also can obtain

$$
\begin{aligned}
u_{i}^{\prime} & \\
= & \frac{2\left(u_{i}-u_{i-1}\right)}{h_{i}} \\
& \quad-\frac{\left(u_{i}-u_{i-1}\right) W_{i}+(r+1 / \Delta t) h_{i}^{2} u_{i-1}-h_{i}^{2} \widetilde{L} u\left(\xi_{i}\right)}{h_{i} M_{i}},
\end{aligned}
$$

where

$$
\begin{aligned}
W_{i}= & -\sigma^{2} \xi_{i}^{2}-2(r-d) \xi_{i} h_{i}\left(1-\alpha_{1, i}\right) \\
& +\left(r+\frac{1}{\Delta t}\right) h_{i}^{2}\left(1-\alpha_{1, i}\right)^{2} \\
M_{i}= & -\sigma^{2} \xi_{i}^{2}+(r-d) \xi_{i} h_{i}\left(-1+2 \alpha_{1, i}\right) \\
& -\left(r+\frac{1}{\Delta t}\right) h_{i}^{2} \alpha_{1, i}\left(1-\alpha_{1, i}\right) .
\end{aligned}
$$

Combining (30) with (33), we have the following spline difference scheme

$$
a_{i}^{-} u_{i-1}+a_{i}^{c} u_{i}+a_{i}^{+} u_{i+1}=q_{i}^{-} \widetilde{L} u\left(\xi_{i}\right)+q_{i}^{+} \widetilde{L} u\left(\eta_{i}\right),
$$

where

$$
\begin{aligned}
& a_{i}^{-}=-\frac{Z_{i}}{h_{i} M_{i}}, \\
& a_{i}^{+}=-\frac{Q_{i}}{h_{i+1} P_{i}}, \\
& a_{i}^{c}=\frac{2}{h_{i}}-\left(r+\frac{1}{\Delta t}\right) \frac{h_{i+1}}{P_{i}}+\frac{Q_{i}}{h_{i+1} P_{i}}-\frac{W_{i}}{h_{i} M_{i}}, \\
& q_{i}^{-}=-\frac{h_{i}}{M_{i}}, \\
& q_{i}^{+}=-\frac{h_{i+1}}{P_{i}}, \\
& Z_{i}=-\sigma^{2} \xi_{i}^{2}+2(r-d) \xi_{i} h_{i} \alpha_{1, i}+\left(r+\frac{1}{\Delta t}\right) h_{i}^{2} \alpha_{1, i}^{2} .
\end{aligned}
$$

Two degrees of freedom are provided by parameters $\alpha_{1, i}$ and $\alpha_{2, i}$, which can be used to guarantee that the matrix associated with the discrete operator is an M-matrix. Here we choose $\alpha_{1, i}=\Delta t$ and $\alpha_{2, i}=1-\Delta t$ for $1 \leq i<N$. In the next section we will prove that $a_{i}^{-}<0, a_{i}^{+}<0, q_{i}^{-}>0, q_{i}^{+}>0$ and $a_{i}^{-}+a_{i}^{c}+$ $a_{i}^{+}-q_{i}^{-} / \Delta t-q_{i}^{+} / \Delta t>0$.
Combining (18)-(22) with (35) we can obtain the following fully discretization scheme

$$
\begin{aligned}
L^{N, K} V_{i}^{j} \geq 0, \quad(i, j) \in \widetilde{\Omega}_{h}, \\
V_{i}^{j}-g_{i} \geq 0, \quad(i, j) \in \widetilde{\Omega}_{h}, \\
L^{N, K} V_{i}^{j} \cdot\left[V_{i}^{j}-g_{i}\right]=0, \quad(i, j) \in \widetilde{\Omega}_{h}, \\
V_{i}^{K}=g_{i}, \quad i=0,1, \cdots, N, \\
V_{0}^{j}=E, \\
V_{N}^{j}=0, \quad \\
\quad j=K-1, \cdots, 1,0,
\end{aligned}
$$

where

$$
\begin{aligned}
L^{N, K} V_{i}^{j} \equiv & a_{i}^{-} V_{i-1}^{j}+a_{i}^{c} V_{i}^{j}+a_{i}^{+} V_{i+1}^{j} \\
& -\frac{q_{i}^{-}}{\Delta t}\left[\alpha_{1, i} V_{i-1}^{j+1}+\left(1-\alpha_{1, i}\right) V_{i}^{j+1}\right] \\
& -\frac{q_{i}^{+}}{\Delta t}\left[\alpha_{2, i} V_{i}^{j+1}+\left(1-\alpha_{2, i}\right) V_{i+1}^{j+1}\right] .
\end{aligned}
$$

Here we have used the linear interpolation to get the approximated solutions $V^{j+1}$ at mesh points $\xi_{i}$ and $\eta_{i}$ since we only know the numerical solution on mesh points at $j+1$-th time level.

There exists a unique solution $V$ for the above linear complementarity problem (37)-(41). A detailed discussion of existence and uniqueness of the solution for problem (37)(41) can be found in [18]. Then from the above solution $V$ we can obtain the optimal stopping price which is the maximum asset price such that $V_{i}^{j}=g_{i}$ for each $t_{j}$.

Remark 1. In general the points $x_{i+1 / 2}$ are used as the collocation points $[19,20]$, i.e., $\alpha_{1, i}=\alpha_{2, i}=1 / 2$. But for the problem (18)-(22) the discrete operator with $\alpha_{1, i}=\alpha_{2, i}=1 / 2$ does not satisfy the discrete maximum principle. Hence, in order to apply the discrete maximum principle and barrier function technique to analyze the stability and error estimate we use collocation points $\xi_{i}$ on $\left[x_{i-1}, x_{i}\right]$ and $\eta_{i}$ on $\left[x_{i}, x_{i+1}\right]$ for $1 \leq i<N$.

\section{Analysis of the Method}

The discrete maximum principle, truncation error analysis techniques, and barrier function techniques are used for analysis of the scheme.

Lemma 2 (discrete maximum principle). When $\alpha_{1, i}=\Delta t$ and $\alpha_{2, i}=1-\Delta t$ for $1 \leq i<N$, the operator $L^{N, K}$ on $\Omega^{N \times K}$ satisfies a discrete maximum principle; i.e., if $w_{i}^{j}$ is a mesh function satisfying $w_{0}^{j} \geq 0, w_{N}^{j} \geq 0(0 \leq j<K), w_{i}^{K} \geq 0(0 \leq i \leq N)$ and $L^{N, K} w_{i}^{j} \geq 0(1 \leq i<N, 0 \leq j<K)$, then $w_{i}^{j} \geq 0$ for all $i, j$. 
Proof. When $\alpha_{1, i}=\Delta t$ and $\alpha_{2, i}=1-\Delta t$, it is easy to check that

$$
\begin{aligned}
Z_{i} & <0, \\
M_{i} & <0,
\end{aligned}
$$

and $Q_{i}<0$

for sufficiently small $\Delta t$ and $h$. We also have

$$
\begin{aligned}
P_{i} & <-\sigma^{2} \eta_{i}^{2}+(r-d) \eta_{i} h_{i+1} \\
& =\eta_{i}\left[-\sigma^{2} \eta_{i}+(r-d) h_{i+1}\right] \\
& <\eta_{i}\left[-\sigma^{2} x_{i}+(r-d) h_{i+1}\right] \\
& <\eta_{i}\left[-\sigma^{2} h+(r-d) \frac{\sigma^{2}}{r-d} h\right]=0 .
\end{aligned}
$$

Hence we can conclude that

$$
\begin{aligned}
& a_{i}^{-}<0, \\
& a_{i}^{+}<0, \\
& q_{i}^{-}>0, \\
& q_{i}^{+}>0,
\end{aligned}
$$

and

$$
\begin{aligned}
& a_{i}^{c}=-a_{i}^{-}-a_{i}^{+}+\left(r+\frac{1}{\Delta t}\right) q_{i}^{-}+\left(r+\frac{1}{\Delta t}\right) q_{i}^{+}>0, \\
& a_{i}^{-}+a_{i}^{c}+a_{i}^{+}-\frac{1}{\Delta t}\left(q_{i}^{-}+q_{i}^{+}\right)=r\left(q_{i}^{-}+q_{i}^{+}\right)>0
\end{aligned}
$$

for $1 \leq i<N$ and $0 \leq j<K$. Hence the matrix associated with $L^{N, K}$ is an M-matrix, which reveals that the operator $L^{N, K}$ satisfies the discrete maximum principle [21].

By using Taylor expansions we can get the following bound of the truncation error

$$
\begin{aligned}
\tau_{i}^{j}(v)= & L^{N, K} v_{i}^{j}-q_{i}^{-} L v\left(\xi_{i}, t_{j}\right)-q_{i}^{+} L v\left(\eta_{i}, t_{j}\right) \\
= & p_{0} v\left(x_{i}, t_{j}\right)+p_{1} \frac{\partial v}{\partial x}\left(x_{i}, t_{j}\right)+p_{2} \frac{\partial^{2} v}{\partial x^{2}}\left(x_{i}, t_{j}\right) \\
& +p_{3} \frac{\partial v}{\partial t}\left(x_{i}, t_{j}\right)+\sum_{n=1}^{12} \pi_{i, n}^{j}(v),
\end{aligned}
$$

where $p_{0}=p_{1}=p_{2}=p_{3}=0$ and

$$
\begin{aligned}
& \pi_{i, 1}^{j}(v)=a_{i}^{+} R_{2}\left(x_{i}, x_{i+1}, v_{j}(x)\right), \\
& \pi_{i, 2}^{j}(v)=-a_{i}^{-} R_{2}\left(x_{i}, x_{i-1}, v_{j}(x)\right),
\end{aligned}
$$

$$
\begin{aligned}
& \pi_{i, 3}^{j}(v)=-\frac{1}{2} \sigma^{2} x_{i}^{2} q_{i}^{-} R_{0}\left(x_{i}, \xi_{i}, \frac{d^{2} v_{j}}{d x^{2}}\right), \\
& \pi_{i, 4}^{j}(v)=-\frac{1}{2} \sigma^{2} x_{i}^{2} q_{i}^{+} R_{0}\left(x_{i}, \eta_{i}, \frac{d^{2} v_{j}}{d x^{2}}\right), \\
& \pi_{i, 5}^{j}(v)=-(r-d) x_{i} q_{i}^{-} R_{1}\left(x_{i}, \xi_{i}, \frac{d v_{j}}{d x}\right), \\
& \pi_{i, 6}^{j}(v)=-(r-d) x_{i} q_{i}^{+} R_{1}\left(x_{i}, \eta_{i}, \frac{d v_{j}}{d x}\right), \\
& \pi_{i, 7}^{j}(v)=\left(r+\frac{1}{\Delta t}\right) q_{i}^{-} R_{2}\left(x_{i}, \xi_{i}, v_{j}(x)\right), \\
& \pi_{i, 8}^{j}(v)=\left(r+\frac{1}{\Delta t}\right) q_{i}^{+} R_{2}\left(x_{i}, \eta_{i}, v_{j}(x)\right), \\
& \pi_{i, 9}^{j}(v)=-\frac{q_{i}^{-}}{\Delta t}\left[\alpha_{1, i} R_{1}\left(\xi_{i}, x_{i-1}, v\left(x, t_{j+1}\right)\right)\right. \\
& \left.+\left(1-\alpha_{1, i}\right) R_{1}\left(\xi_{i}, x_{i}, v\left(x, t_{j+1}\right)\right)\right], \\
& \pi_{i, 10}^{j}(v)=-\frac{q_{i}^{-}}{\Delta t}\left[\alpha_{2, i} R_{1}\left(\eta_{i}, x_{i}, v\left(x, t_{j+1}\right)\right)\right. \\
& \left.+\left(1-\alpha_{2, i}\right) R_{1}\left(\eta_{i}, x_{i+1}, v\left(x, t_{j+1}\right)\right)\right], \\
& \pi_{i, 11}^{j}(v)=-\frac{q_{i}^{-}}{\Delta t} R_{1}\left(x_{i}, \xi_{i}, v\left(x, t_{j}\right)\right)-\frac{q_{i}^{+}}{\Delta t} \\
& \cdot R_{1}\left(x_{i}, \eta_{i}, v\left(x, t_{j}\right)\right) \\
& \pi_{i, 12}^{j}(v)=-q_{i}^{-} \Delta t R_{1}\left(t_{j}, t_{j+1}, v\left(\xi_{i}, t\right)\right) \\
& -q_{i}^{+} \Delta t R_{1}\left(t_{j}, t_{j+1}, v\left(\eta_{i}, t\right)\right), \\
& R_{n}(x, z, u)=\frac{(z-x)^{n+1}}{(n+1) !} u^{(n+1)}(s), \quad s \in(x, z) .
\end{aligned}
$$

Thus, when $\alpha_{1, i}=\Delta t$ and $\alpha_{2, i}=1-\Delta t$ for $1 \leq i<N$, from (47) we have

$$
\left|\tau_{i}^{j}\right| \leq C\left(q_{i}^{-}+q_{i}^{+}\right)\left(h^{2}+\Delta t\right) \quad 1 \leq i<N, 0 \leq j<K,
$$

where $C$ is a positive constant independent of the mesh.

The following theorem is our main result for the quadratic spline collocation scheme.

Theorem 3. Let $v(x, t)$ be the solution of problem (9)-(13) and $V_{i}^{j}$ the solution of problem (37)-(41). Then if we set $\alpha_{1, i}=\Delta t$ and $\alpha_{2, i}=1-\Delta t$ for $1 \leq i<N$, there exists a positive constant $C$ independent of the mesh such that

$$
\left|v\left(x_{i}, t_{j}\right)-V_{i}^{j}\right| \leq C\left(h^{2}+\Delta t\right),
$$

$0 \leq i \leq N, 0 \leq j \leq K$. 
Proof. The error estimate will be derived by applying the maximum principle to the discrete linear complementarity problem in two mesh sets as that in [22]. Set

$$
\begin{aligned}
\bar{\Omega}_{h} & =\{(i, j) \mid 0 \leq i \leq N, 0 \leq j \leq K\}, \\
\widetilde{\Omega}_{h} & =\{(i, j) \mid 1 \leq i \leq N-1,0 \leq j \leq K-1\}, \\
\Omega^{(1)} & =\left\{(i, j) \in \widetilde{\Omega}_{h} \mid v\left(x_{i}, t_{j}\right)=g_{i}\right\}, \\
\Omega^{(2)} & =\widetilde{\Omega}_{h} \backslash \Omega^{(1)}, \\
\partial \Omega_{h} & =\bar{\Omega}_{h} \backslash \widetilde{\Omega}_{h} .
\end{aligned}
$$

From (9)-(13) we have

$$
\begin{aligned}
& L v\left(x_{i}, t_{j}\right) \geq 0, \quad(i, j) \in \Omega^{(1)}, \\
& L v\left(x_{i}, t_{j}\right)=0, \quad(i, j) \in \Omega^{(2)} .
\end{aligned}
$$

Let

$$
\begin{aligned}
& \Omega_{h}^{(1)}=\left\{(i, j) \in \widetilde{\Omega}_{h} \mid V_{i}^{j}=g_{i}\right\}, \\
& \Omega_{h}^{(2)}=\widetilde{\Omega}_{h} \backslash \Omega_{h}^{(1)} .
\end{aligned}
$$

It is easy to check that

$$
L^{N, K} V_{i}^{j}=0, \quad(i, j) \in \Omega_{h}^{(2)} .
$$

Define the barrier function

$$
\Phi_{i}^{j}=C\left(h^{2}+\Delta t\right)>0, \quad(i, j) \in \widetilde{\Omega}_{h},
$$

where $C$ is a sufficiently large constant independent of $h$ and $\Delta t$.

For $(i, j) \in \Omega_{h}^{(2)}$ we have

$$
\begin{aligned}
& L^{N, K}\left(v\left(x_{i}, t_{j}\right)-V_{i}^{j}+\Phi_{i}^{j}\right)=L^{N, K} v\left(x_{i}, t_{j}\right) \\
& +L^{N, K} \Phi_{i}^{j}=\left[L^{N, K} v\left(x_{i}, t_{j}\right)-q_{i}^{-} L v\left(\xi_{i}, t_{j}\right)\right. \\
& \left.-q_{i}^{+} L v\left(\eta_{i}, t_{j}\right)+L^{N, K} \Phi_{i}^{j}\right]+q_{i}^{-} L v\left(\xi_{i}, t_{j}\right) \\
& +q_{i}^{+} L v\left(\eta_{i}, t_{j}\right) \geq 0
\end{aligned}
$$

by using $L v\left(\xi_{i}, t_{j}\right) \geq 0, L v\left(\eta_{i}, t_{j}\right) \geq 0$ and (49), (54), and (55). On the "boundary" of the $\Omega_{h}^{(2)}$ we have

$$
\begin{array}{r}
v\left(x_{i}, t_{j}\right)-V_{i}^{j}+\Phi_{i}^{j}=v\left(x_{i}, t_{j}\right)-g_{i}+\Phi_{i}^{j} \geq 0 \\
\qquad \text { for }(i, j) \in \Omega_{h}^{(1)},
\end{array}
$$

and

$$
v\left(x_{i}, t_{j}\right)-V_{i}^{j}+\Phi_{i}^{j}=\Phi_{i}^{j} \geq 0 \quad \text { for }(i, j) \in \partial \Omega_{h} .
$$

Then we have

$$
v\left(x_{i}, t_{j}\right)-V_{i}^{j}+\Phi_{i}^{j} \geq 0, \quad(i, j) \in \Omega_{h}^{(2)}
$$

by using the maximum principle (Lemma 2) to $\Omega_{h}^{(2)}$. Hence, we have

$$
v\left(x_{i}, t_{j}\right)-V_{i}^{j}+\Phi_{i}^{j} \geq 0, \quad(i, j) \in \bar{\Omega}_{h} .
$$

For $(i, j) \in \Omega^{(2)}, L v\left(x_{i}, t_{j}\right)=0$, but $L^{N, K} V_{i}^{j} \geq 0$; thus for the sufficiently large constant $C$ we have

$$
\begin{aligned}
L^{N, K} & \left(v\left(x_{i}, t_{j}\right)-V_{i}^{j}-\Phi_{i}^{j}\right)=\left[L^{N, K} v\left(x_{i}, t_{j}\right)\right. \\
& \left.-q_{i}^{-} L v\left(\xi_{i}, t_{j}\right)-q_{i}^{+} L v\left(\eta_{i}, t_{j}\right)-L^{N, K} \Phi_{i}^{j}\right] \\
& +q_{i}^{-} L v\left(\xi_{i}, t_{j}\right)+q_{i}^{+} L v\left(\eta_{i}, t_{j}\right)-L^{N, K} V_{i}^{j} \\
& \leq\left\{L^{N, K} v\left(x_{i}, t_{j}\right)-q_{i}^{-} L v\left(\xi_{i}, t_{j}\right)-q_{i}^{+} L v\left(\eta_{i}, t_{j}\right)\right. \\
& -L^{N, K} \Phi_{i}^{j} \\
& \left.+\left[q_{i}^{-} \alpha_{1, i}\left(1-\alpha_{1, i}\right)+q_{i}^{+} \alpha_{2, i}\left(1-\alpha_{2, i}\right)\right] O\left(h^{2}\right)\right\} \\
& +q_{i}^{-}\left[\alpha_{1, i} L v\left(x_{i-1}, t_{j}\right)+\left(1-\alpha_{1, i}\right) L v\left(x_{i}, t_{j}\right)\right] \\
& +q_{i}^{+}\left[\alpha_{2, i} L v\left(x_{i}, t_{j}\right)+\left(1-\alpha_{2, i}\right) L v\left(x_{i+1}, t_{j}\right)\right] \\
& -L^{N, K} V_{i}^{j} \leq\left\{L^{N, K} v\left(x_{i}, t_{j}\right)-q_{i}^{-} L v\left(\xi_{i}, t_{j}\right)\right. \\
& -q_{i}^{+} L v\left(\eta_{i}, t_{j}\right)-L^{N, K} \Phi_{i}^{j} \\
& +\left[q_{i}^{-} \alpha_{1, i}\left(1-\alpha_{1, i}\right)+q_{i}^{+} \alpha_{2, i}\left(1-\alpha_{2, i}\right)\right] O\left(h^{2}\right) \\
& \left.+\left(q_{i}^{-}+q_{i}^{+}\right) O(\Delta t)\right\}-L^{N, K} V_{i}^{j} \leq 0,
\end{aligned}
$$

where we have used $\alpha_{1, i}=1-\alpha_{2, i}=\Delta t$ and (49). On the "boundary" of the $\Omega^{(2)}$ we have

$$
\begin{aligned}
v\left(x_{i}, t_{j}\right)-V_{i}^{j}-\Phi_{i}^{j}=g_{i}-V_{i}^{j}-\Phi_{i}^{j} & \leq 0 \\
& \text { for }(i, j) \in \Omega^{(1)},
\end{aligned}
$$

and

$$
v\left(x_{i}, t_{j}\right)-V_{i}^{j}-\Phi_{i}^{j}=-\Phi_{i}^{j} \leq 0 \quad \text { for }(i, j) \in \partial \Omega_{h} .
$$

Then we have

$$
v\left(x_{i}, t_{j}\right)-V_{i}^{j}-\Phi_{i}^{j} \leq 0, \quad(i, j) \in \Omega^{(2)}
$$

by using the maximum principle (Lemma 2) to $\Omega^{(2)}$. Hence

$$
v\left(x_{i}, t_{j}\right)-V_{i}^{j}-\Phi_{i}^{j} \leq 0, \quad(i, j) \in \bar{\Omega}_{h} .
$$

Combining (60) with (65) we have

$$
\max _{(i, j) \in \bar{\Omega}_{h}}\left|v\left(x_{i}, t_{j}\right)-V_{i}^{j}\right| \leq \max _{(i, j) \in \bar{\Omega}_{h}} \Phi_{i}^{j} \leq C\left(h^{2}+\Delta t\right),
$$

which completes the proof. 


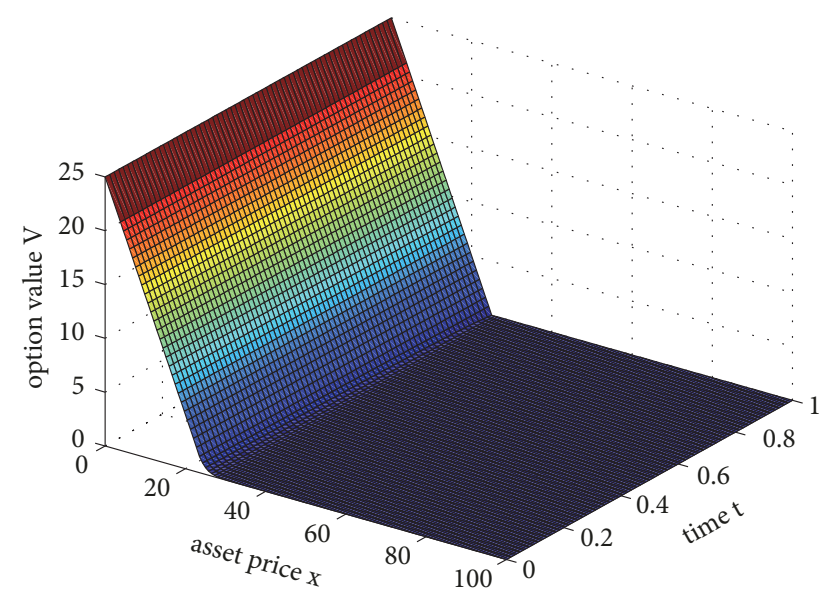

Figure 1: Computed option value $V$ for Example 1.

\section{Numerical Experiments}

In this section we perform the numerical experiments to illustrate the applicability and accuracy of the method obtained in the preceding section. Errors and convergence rates for the spline difference scheme are presented for two examples.

Example 1. Compute the value of an American put option with $\sigma=0.1, r=0.08, d=0.03, T=1, E=25$, and $X=100$.

Example 2. Compute the value of an American put option with $\sigma=0.5, r=0.1, d=0.02, T=1, E=25$, and $X=100$.

The projection scheme [23] is used to solve the linear inequality system (37)-(41). Assume that $V^{j+1}$ are known; then we first solve the following discretization problem for $\left\{V^{j+1 / 2}\right\}$ :

$$
\begin{aligned}
L^{N, K} V_{i}^{j+1 / 2} & =0, \quad i=1, \cdots, N-1, \\
V_{0}^{j+1 / 2} & =E, \\
V_{N}^{j+1 / 2} & =0 .
\end{aligned}
$$

Next we set

$$
V_{i}^{j}=\max \left\{V_{i}^{j+1 / 2}, g_{i}\right\}, \quad i=0,1, \cdots, N,
$$

which ensure that (38) and (39) are satisfied.

The computed option values $V$ and the constraints $V$ $g$ with $N=128$ and $K=64$ are plotted in Figures 1-4 for Examples 1 and 2, respectively.

Since the exact solutions of our examples are not available, the quadratic spline approximated solution of $N=2048, K=$ 4096 is used as the exact solution denoted by $\widetilde{V}(x, t)$. The error estimates for different $N$ at $t=0$ are presented. The accuracy in the discrete maximum-norm,

$$
e^{N, K}=\max _{i, j}\left|V_{i, 0}^{N, K}-\widetilde{V}\left(x_{i}, 0\right)\right|,
$$

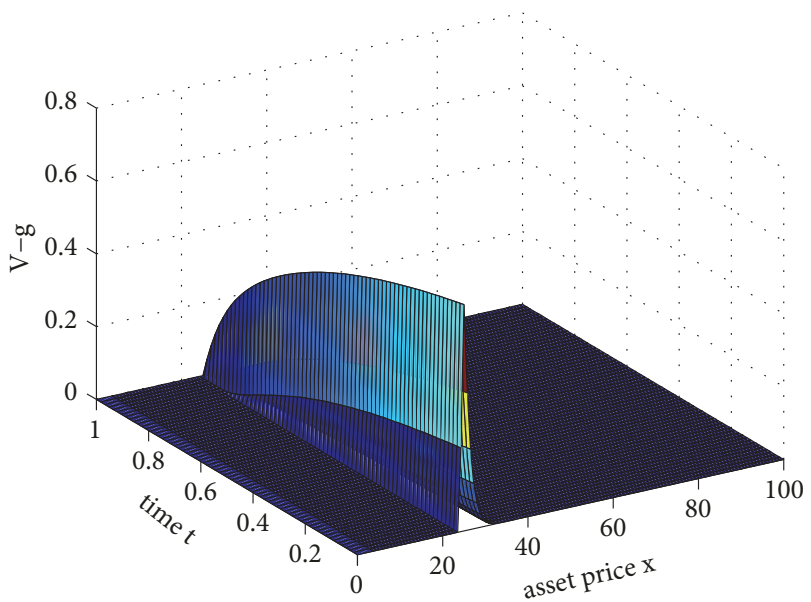

Figure 2: The constraint $V-g$ for Example 1.

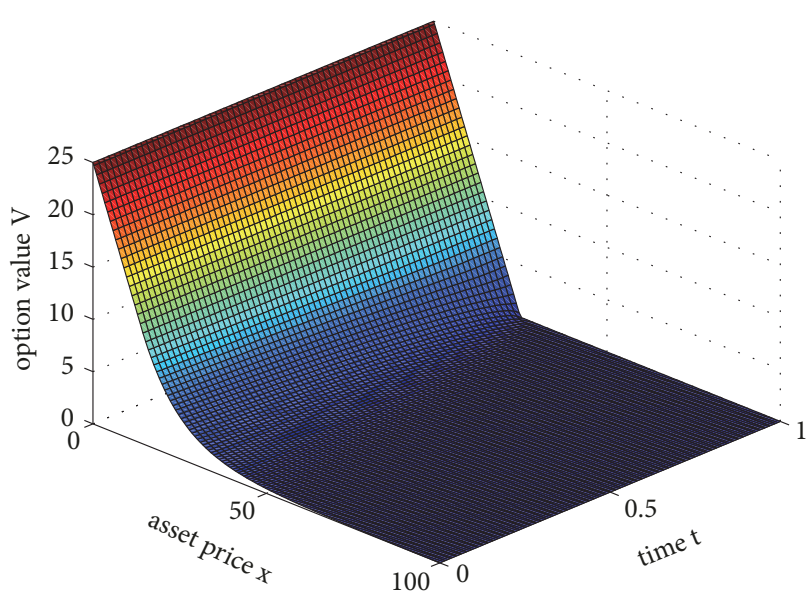

Figure 3: Computed option value $V$ for Example 2.

TABLE 1: Error estimates and convergence rates for Example 1.

\begin{tabular}{lccc}
\hline$K$ & $N$ & error & rate \\
\hline 1024 & 128 & $2.3532 \mathrm{e}-2$ & 2.009 \\
& 256 & $5.8452 \mathrm{e}-3$ & 1.914 \\
& 512 & $1.5506 \mathrm{e}-3$ & 2.342 \\
& 1024 & $3.0592 \mathrm{e}-4$ & - \\
\hline
\end{tabular}

and the convergence rate

$$
R^{N, K}=\log _{2}\left(\frac{e^{N, K}}{e^{2 N, K}}\right)
$$

are computed. The error estimates and convergence rates for Examples 1 and 2 are listed in Tables 1 and 2, respectively.

From Figures 1 and 3 we can conclude that the numerical solutions are nonoscillatory, and from Figures 2 and 4 we also can conclude that the numerical solutions satisfy the constraint conditions (38) and (39). Tables 1 and 2 show that 


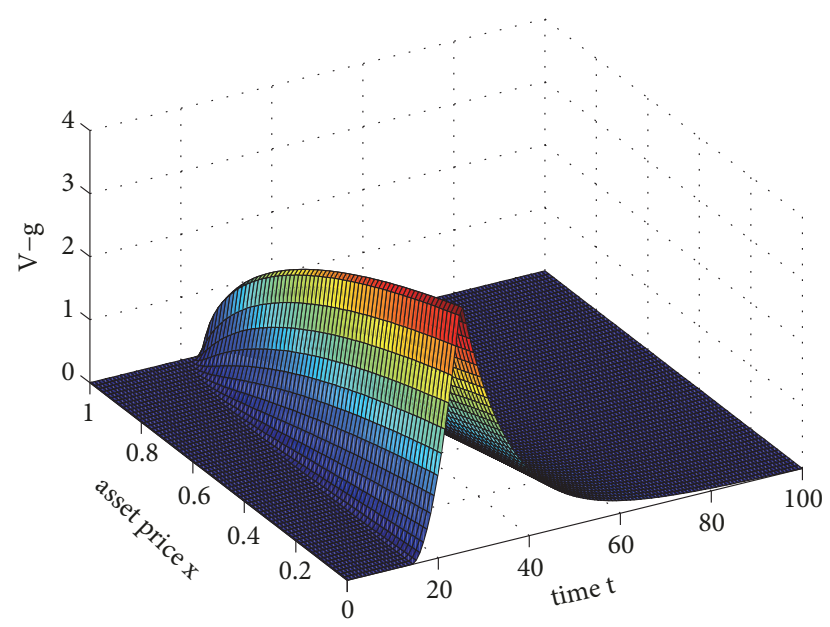

Figure 4: The constraint $V-g$ for Example 2.

TABLE 2: Error estimates and convergence rates for Example 2.

\begin{tabular}{lccc}
\hline$K$ & $N$ & error & rate \\
\hline 1024 & 128 & $8.6028 \mathrm{e}-3$ & 2.031 \\
& 256 & $2.1045 \mathrm{e}-3$ & 2.097 \\
& 512 & $4.9176 \mathrm{e}-4$ & 2.451 \\
& 1024 & $8.9918 \mathrm{e}-5$ & - \\
\hline
\end{tabular}

$R^{N, K}$ is close to 2 for sufficiently large $N$. These results support the convergence estimate of Theorem 3.

Finally, our scheme is compared with the binomial method, analytic approximation method, and compact finite difference methods. The numerical example and numerical results in Zhao [15] are used. The parameters for the American put option are $\sigma=0.3, T=1, r=0.04, d=0.02$, and $E=$ 100. The results for the binomial method are obtained with the time mesh size $\Delta t=0.01$. The results for the analytical approximation method are obtained with the time mesh size $\Delta t=0.02$. The results for the compact finite difference methods 1, 2, and 3 are obtained with the spatial mesh size $h=0.02$ and the time mesh size $\Delta t=0.0005$. For our spline collocation scheme we use the time mesh size $\Delta t=0.0005$ and $N=20000$ mesh points for the spatial discretization which has almost same number of mesh points as that of the compact methods. The true option values are obtained with the trinomial method using the time mesh size $\Delta t=0.00005$. Table 3 shows that our scheme is more accurate than other methods.

As discussed above, the optimal stopping boundary can be obtained as the maximum asset price such that $V_{i}^{j}=g_{i}$ for each $t_{j}$. We plot the optimal stopping boundary for the two examples with $N=K=2048$ in Figures 5 and 6, respectively.

\section{Conclusion and Discussion}

In this paper the linear complementarity problem arising from American put option pricing is treated by a quadratic spline collocation method on a piecewise uniform mesh. The main advantage of the spline collocation method examined

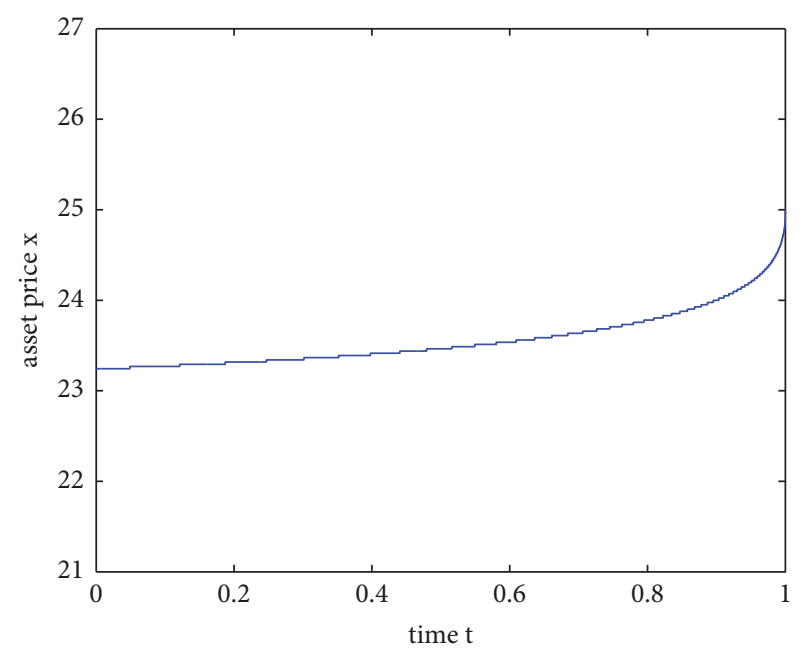

FIGURE 5: The optimal stopping boundary for Example 1.

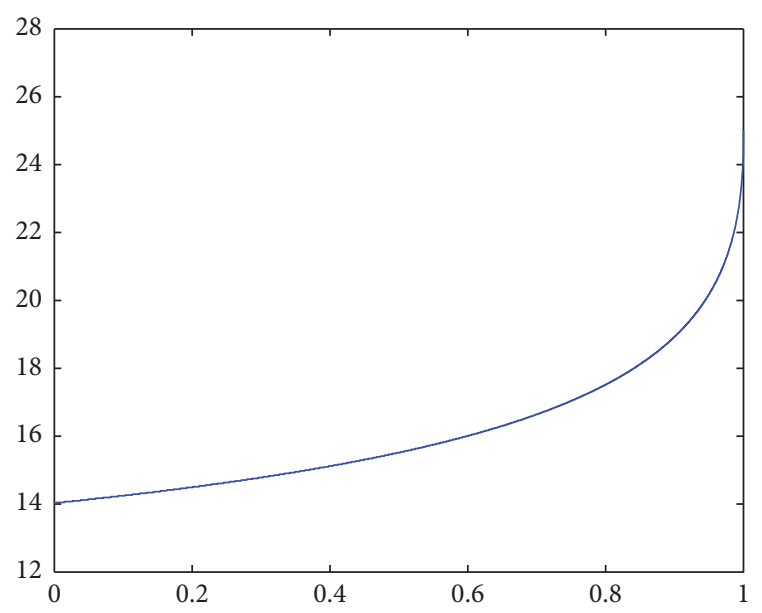

FIGURE 6: The optimal stopping boundary for Example 2.

in the present paper lies in the possibility of simple and direct constructing of a continuous approximate solution and normalized flux between the mesh points. The suitable choice of collocation points guarantees that the spline difference operator on the piecewise uniform mesh satisfies the discrete maximum principle. Hence the scheme is maximum-norm stable. The error estimation is derived by applying the maximum principle to the discrete linear complementarity problem in two mesh sets. It is proved that the scheme is second-order convergent with respect to the spatial variable and first-order convergent with respect to the time variable. Numerical results demonstrate that the scheme is stable and accurate.

For the time discretization, although the Crank-Nicolson scheme can be used to improve the truncation error scheme, the discretization scheme does not satisfy the maximum principle without additional constraints about the time sizes, which leads to the difficulty in the derivation of the error estimation for the linear complementarity problem under the difference schemes. In future we plan to overcome this 


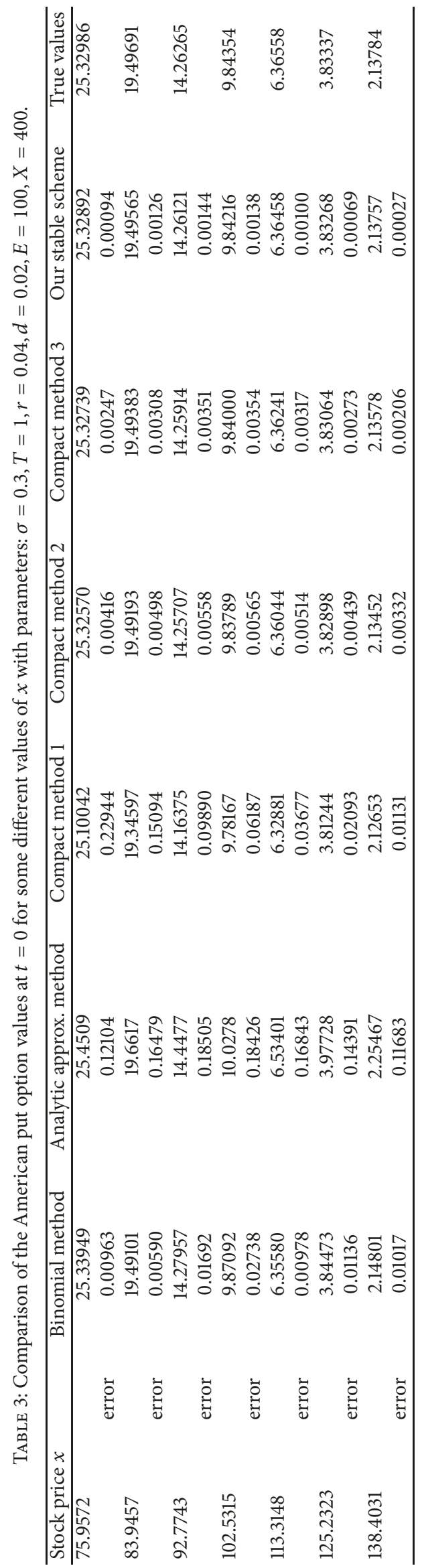


difficulty in the error analysis for the Crank-Nicolson scheme in discretizing the linear complementarity problem.

Since the probability distribution of realized asset returns may exhibit features such as heavy tails, volatility clustering, and volatility smile, two different classes of models have been studied in the finance literature: the jump-diffusion models $[24,25]$ and the stochastic volatility models $[26,27]$. In future we extend this technique to construct spline collocation schemes and study the stability and error analysis of these schemes for the jump-diffusion models and the stochastic volatility models.

\section{Data Availability}

The data used to support the findings of this study are included within the article.

\section{Conflicts of Interest}

The authors declare that they have no conflicts of interest.

\section{Acknowledgments}

The work was supported by Major Humanities and Social Sciences Projects in Colleges and Universities of Zhejiang Province of China (Grant no. 2018GH020).

\section{References}

[1] P. Wilmott, J. Dewynne, and S. Howison, Option Pricing: Mathematical Models and Computation, Oxford Financial Press, Oxford, 1993.

[2] M. H. Khabir and K. C. Patidar, "Spline approximation method to solve an option pricing problem," Journal of Difference Equations and Applications, vol. 18, no. 11, pp. 1801-1816, 2012.

[3] M. K. Kadalbajoo, L. P. Tripathi, and P. Arora, "A robust nonuniform B-spline collocation method for solving the generalized BLAck-Scholes equation," IMA Journal of Numerical Analysis (IMAJNA), vol. 34, no. 1, pp. 252-278, 2014.

[4] M. K. Kadalbajoo, L. P. Tripathi, and A. Kumar, "A cubic Bspline collocation method for a numerical solution of the generalized BLAck-Scholes equation," Mathematical and Computer Modelling, vol. 55, no. 3-4, pp. 1483-1505, 2012.

[5] R. Mohammadi, "Quintic B-spline collocation approach for solving generalized BLAck-Scholes equation governing option pricing," Computers \& Mathematics with Applications. An International Journal, vol. 69, no. 8, pp. 777-797, 2015.

[6] C. C. Christara and N. C. Leung, "Option pricing in jump diffusion models with quadratic spline collocation," Applied Mathematics and Computation, vol. 279, pp. 28-42, 2016.

[7] J. Rashidinia and S. Jamalzadeh, "Modified B-spline collocation approach for pricing American style ASIan options," Mediterranean Journal of Mathematics, vol. 14, no. 3, Art. 111, 17 pages, 2017.

[8] K. Surla, L. Teofanov, and Z. Uzelac, "A robust layer-resolving spline collocation method for a convection-diffusion problem," Applied Mathematics and Computation, vol. 208, no. 1, pp. 7689, 2009.

[9] K. Surla, Z. Uzelac, and L. Teofanov, "The discrete minimum principle for quadratic spline discretization of a singularly perturbed problem," Mathematics and Computers in Simulation, vol. 79, no. 8, pp. 2490-2505, 2009.

[10] J. Huang and J. Pang, "Option pricing and linear complementarity," The Journal of Computational Finance, vol. 2, no. 1, pp. 31-60, 1998.

[11] D. Jeong, S. Seo, H. Hwang, D. Lee, Y. Choi, and J. Kim, "Accuracy, robustness, and efficiency of the linear boundary condition for the Black-Scholes equations," Discrete Dynamics in Nature and Society, vol. 2015, Article ID 359028, 10 pages, 2015.

[12] R. Kangro and R. Nicolaides, "Far field boundary conditions for Black-Scholes equations," SIAM Journal on Numerical Analysis, vol. 38, no. 4, pp. 1357-1368, 2000.

[13] E. S. Schwartz, "The valuation of warrants: Implementing a new approach," Journal of Financial Economics, vol. 4, no. 1, pp. 7993, 1977.

[14] D. Y. Tangman, A. Gopaul, and M. Bhuruth, "Numerical pricing of options using high-order compact finite difference schemes," Journal of Computational and Applied Mathematics, vol. 218, no. 2, pp. 270-280, 2008.

[15] J. Zhao, M. Davison, and R. M. Corless, "Compact finite difference method for American option pricing," Journal of Computational and Applied Mathematics, vol. 206, no. 1, pp. 306-321, 2007.

[16] Z. Cen and A. Le, "A robust and accurate finite difference method for a generalized Black-Scholes equation," Journal of Computational and Applied Mathematics, vol. 235, no. 13, pp. 3728-2733, 2011.

[17] D. Jeong, M. Yoo, and J. Kim, "Accurate and efficient computations of the Greeks for options near expiry using the BlackScholes equations," Discrete Dynamics in Nature and Society, vol. 2016, Article ID 1586786, 12 pages, 2016.

[18] D. Goeleven, "A uniqueness theorem for the generalized-order linear complementary problem associated with M-matrices," Linear Algebra and its Applications, vol. 235, pp. 221-227, 1996.

[19] K. Surla and Z. Uzelac, "A uniformly accurate spline collocation method for a normalized flux," Journal of Computational and Applied Mathematics, vol. 166, no. 1, pp. 291-305, 2004.

[20] L. Teofanov and Z. Uzelac, "Family of quadratic spline difference schemes for a convection-diffusion problem," International Journal of Computer Mathematics, vol. 84, no. 1, pp. 33-50, 2007.

[21] R. B. Kellogg and A. Tsan, "Analysis of some difference approximations for a singular perturbation problem without turning points," Mathematics of Computation, vol. 32, no. 144, pp. 10251039, 1978.

[22] X.-1. Cheng and L. Xue, "On the error estimate of finite difference method for the obstacle problem," Applied Mathematics and Computation, vol. 183, no. 1, pp. 416-422, 2006.

[23] R. Glowinski, J. L. Lions, and R. Tremolieres, Numerical Analysis of Variational Inequalities, North-Holland, Amsterdam, The Netherlands, 1981.

[24] L. Feng and V. Linetsky, "Pricing options in jump-diffusion models: an extrapolation approach," Operations Research, vol. 56, no. 2, pp. 304-325, 2008.

[25] G. Tour and D. Y. Tangman, "Cubic B-spline collocation method for pricing path dependent options," Lecture Notes in Computer Science (including subseries Lecture Notes in Artificial Intelligence and Lecture Notes in Bioinformatics): Preface, vol. 8584, no. 6, pp. 372-385, 2014. 
[26] B. Düring, M. Fournié, and C. Heuer, "High-order compact finite difference schemes for option pricing in stochastic volatility models on non-uniform grids," Journal of Computational and Applied Mathematics, vol. 271, pp. 247-266, 2014.

[27] C. Guardasoni and S. Sanfelici, "Fast numerical pricing of barrier options under stochastic volatility and jumps," SIAM Journal on Applied Mathematics, vol. 76, no. 1, pp. 27-57, 2016. 


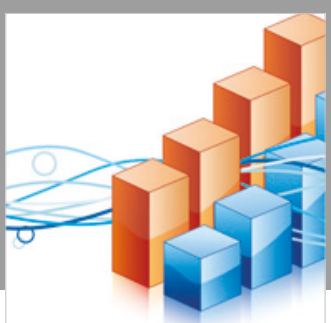

Advances in

Operations Research

\section{-n-m}
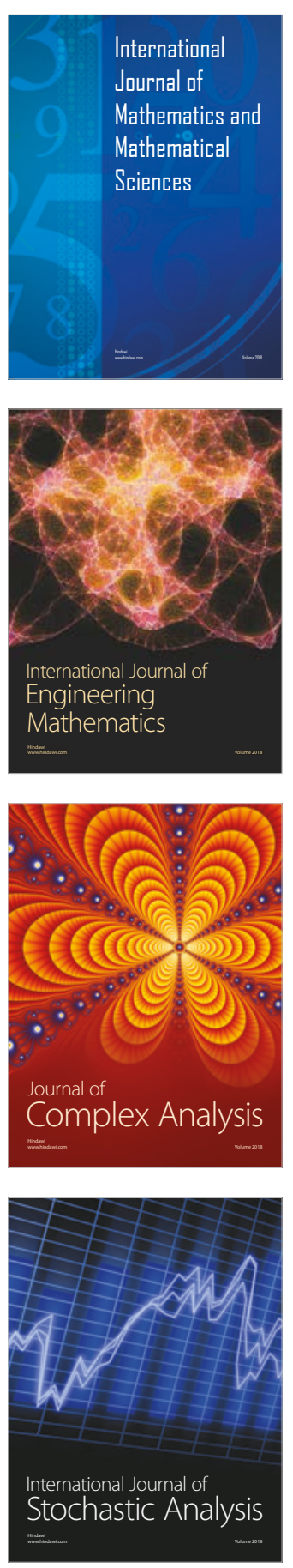
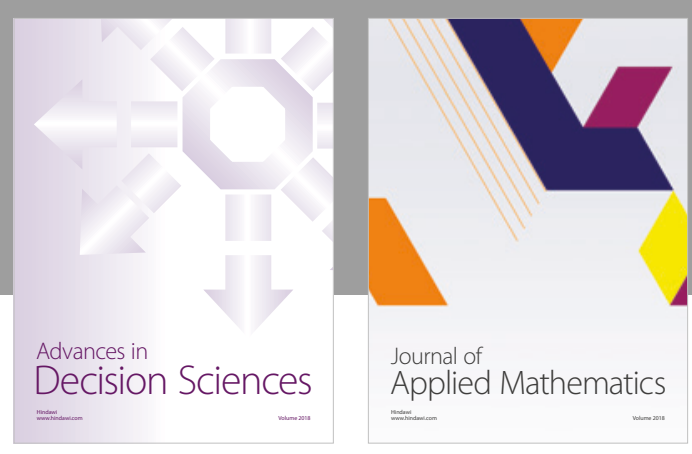

Journal of

Applied Mathematics
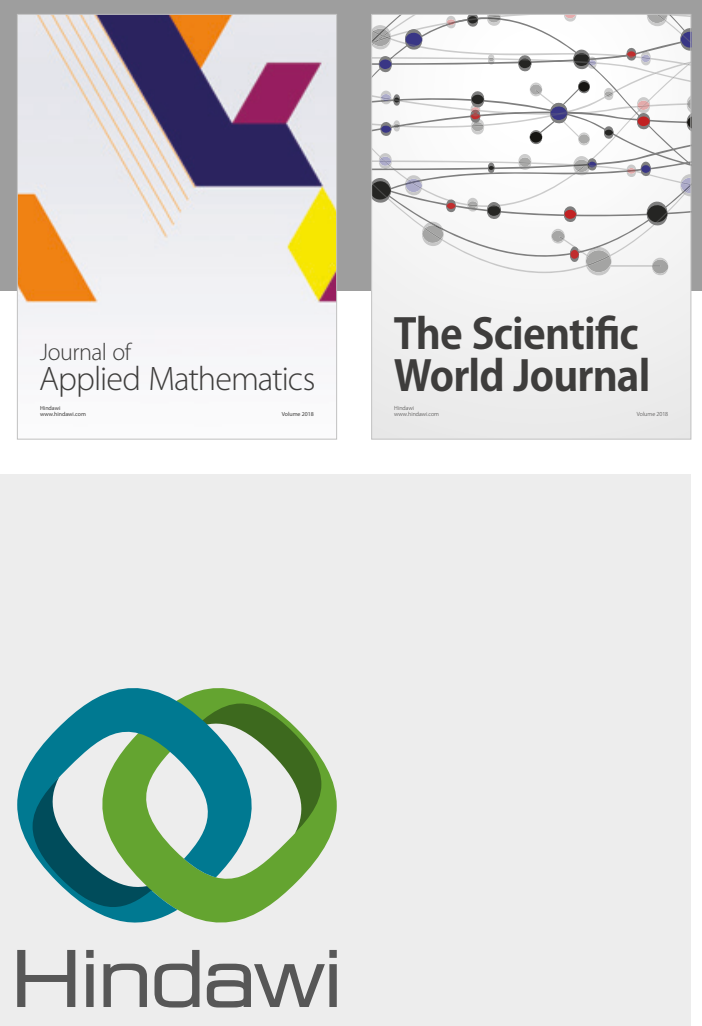

Submit your manuscripts at

www.hindawi.com

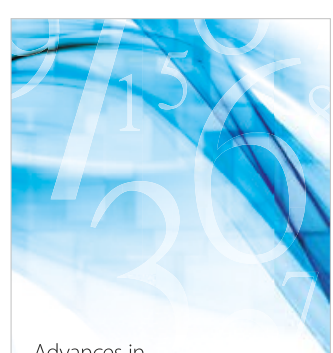

Advances in
Numerical Analysis
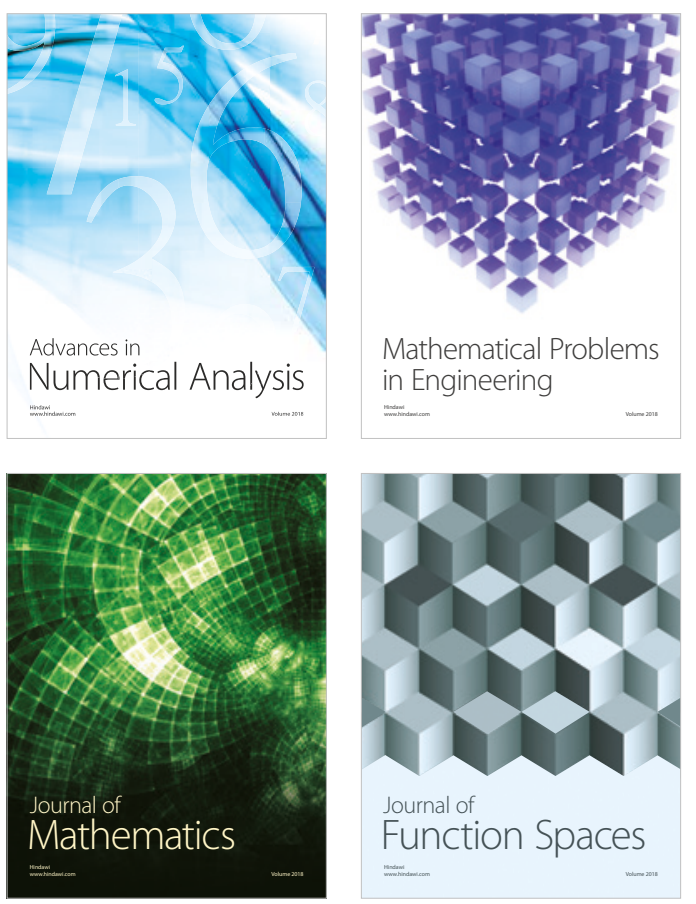

Mathematical Problems in Engineering

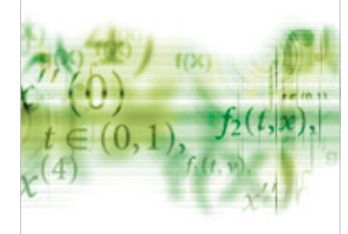

International Journal of

Differential Equations

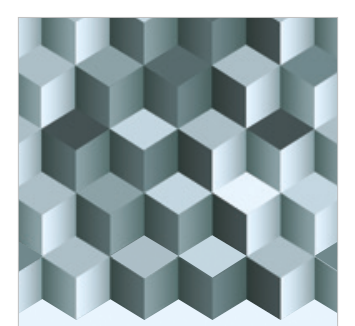

Journal of

Function Spaces

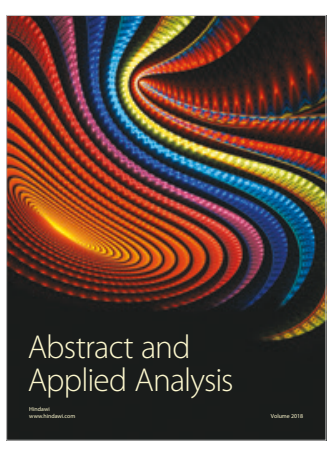

The Scientific

World Journal

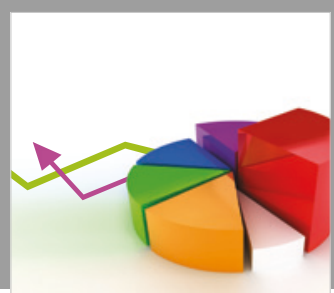

Journal of

Probability and Statistics
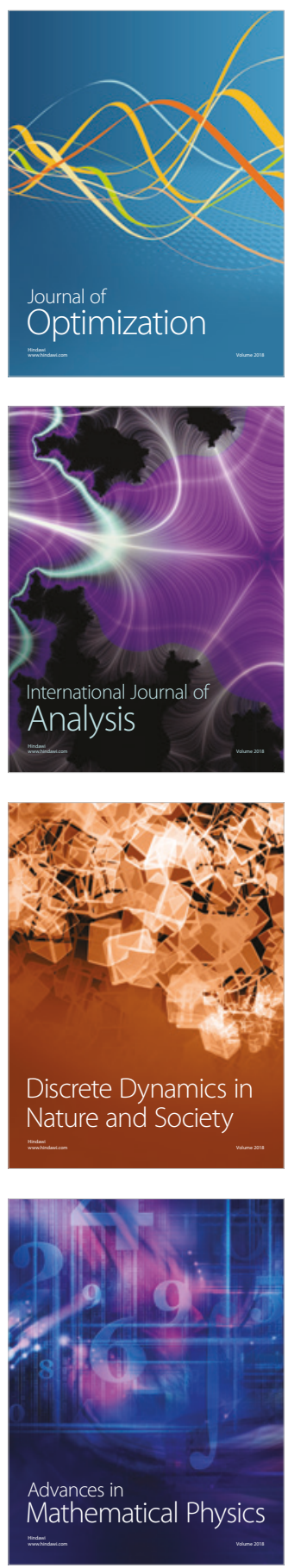